\title{
CORONAVIRUS
}

\section{COVID-19 vaccine side effects: The positives about feeling bad}

\author{
Jonathan Sprent ${ }^{\mathbf{t}^{*}}$ and Cecile King ${ }^{2^{*}}$ \\ ${ }^{1}$ Garvan Institute of Medical Research, Sydney, NSW 2010, Australia \\ ${ }^{2}$ School of Biotechnology and Biomolecular Sciences, University of New South Wales, Sydney, NSW 2052, Australia \\ *Corresponding author. Email: j.sprent@garvan.org.au and c.king@unsw.edu.au
}

The side effects of SARS-CoV-2 vaccines are often troubling but may merely reflect transient production of type I interferons, a normal physiological response to contact with invading microorganisms.

The development of multiple vaccines against SARS-CoV-2 virus, the cause of coronavirus disease 2019 (COVID-19), within one year of the epidemic is unprecedented and an immense accomplishment. The efficacy of many developed vaccines exceeded expectations, and there are high hopes that the epidemic will soon be in the past. Yet several challenges remain. Vaccinations are far from complete in developed nations and have barely begun in many developing nations, suggesting that achieving world-wide herd immunity against the virus may take several years. There is also the growing problem of vaccine hesitancy, especially in the young who generally cope well with COVID-19, with minimal or even no symptoms. In addition, it is well documented that COVID-19 vaccines can have substantial side effects; indeed, fear of these side effects may approach that of SARS-CoV-2 infection itself in some populations. Therefore, what are the side effects of COVID-19 vaccines - and could they paradoxically be beneficial?

In keeping with their rapid development and production, the mRNA-based vaccines of Pfizer and Moderna have received the most attention with regard to the side effects of vaccination $(1,2)$. As with other vaccines, these effects can on rare occasion be the result of delayed-onset, local allergic reactions. In the vast majority of cases, however, the major complaint is a combination of fever, headache, myalgia and general malaise, affecting $\sim 60 \%$ of recipients after the second dose of the vaccines. These symptoms can be troubling and have been the subject of comment in the press and in top scientific journals. Yet, other than vague reference to an ongoing immune response, the actual cause of the side effects has received almost no attention. So what $i s$ the cause of these effects? As discussed here, most of the symptoms can likely be attributed simply to exuberant production of a cytokine that plays a vital role in potentiating early stages of the immune response, namely type I interferon (IFN-I).

The features and functions of IFN-I have been considered elsewhere $(3,4)$. In brief, IFN-I comprises a mixture of IFN$\beta$, multiple subtypes of IFN- $\alpha$, and several other IFNs. IFN-I together with closely related IFN-III (IFN- $\lambda$ ) are produced soon after contact with pathogens and have powerful antiviral effects, acting throughout the body for IFN-I and within the respiratory system for IFN-III. These effects suppress local viral replication and thereby prevent dissemination of virus elsewhere. IFN-I is produced primarily by macrophages and dendritic cells (DC), including both conventional and plasmacytoid DC, and is elicited via interaction with pathogen-associated molecular patterns (PAMPs) expressed by the viral or bacterial pathogen concerned (Fig. 1). PAMPS then interact with complementary pattern recognition receptors (PRRs) expressed by DC, including toll-like receptors (TLRs) and members of the RIG-I-like receptor family; for mRNAbased vaccines, the PAMP (mRNA) is recognized by multiple PRRs, namely TLR7,8, and 9, RIG-I and MDA5.

The receptor for IFN-I, IFNAR, is expressed by all nucleated cells, and contact with its ligand induces a complex series of intracellular signaling events leading to production of a wide range of cytokines and other mediators that antagonize the pathogen concerned. In particular, early production of IFN-I is crucial for producing an optimal immune response. IFN-I induces activation of DC and thereby enables these cells to present antigen to naïve $\mathrm{CD} 4^{+}$and $\mathrm{CD} 8^{+} \mathrm{T}$ cells (Fig. 1); activated $\mathrm{CD}^{+}$cells then stimulate specific antibody production by $\mathrm{B}$ cells, whereas $\mathrm{CD} 8^{+}$cells differentiate into cytolytic effector cells. For these two T cell subsets, IFN-I acts in part by improving the immunogenicity of DC, particularly by elevating the surface expression of molecules that costimulate $\mathrm{T}$ cell activation. In addition, IFN-I has a direct stimulatory effect on $\mathrm{T}$ cells, promoting optimal expansion of these cells and formation of long-lived memory cells, both for CD4 ${ }^{+}$ and $\mathrm{CD} 8^{+} \mathrm{T}$ cells.

For highly pathogenic viruses, IFN-I generation can sometimes be excessive and lead to a pathogenic "cytokine storm" 
$(3,4)$. This is likely not the case for COVID-19, however, because SARS-CoV-2 antagonizes IFN-I production and leads to below-normal levels of IFN-I, especially IFN- $\beta$, in the blood even in patients with severe disease (5). Hence, it seems unlikely that the excessive production of pro-inflammatory cytokines such as IL-6 detected during severe COVID-19 disease is IFN-I-mediated. Moreover, it is notable that patients with severe disease often have high levels of autoantibodies to IFN-I (6). This finding implies that disease severity in these patients is associated with a paucity of IFN-I during the early stages of infection. In support of this notion, there is accumulating evidence that infusion of exogenous IFN-I is efficacious when given early in disease and also when administered prophylactically, especially intranasally. The important issue of whether IFN-I therapy given late in disease exacerbates pathogenesis or is merely ineffective at this stage is still unclear. Currently, however, in contrast to other viruses, there is little or no evidence that IFN-I has a pathogenic effect during SARS-CoV-2 infection.

To date, we have been unable to locate direct evidence on IFN-I production after vaccination against SARS-CoV-2 infection. This is more than likely, however, given that other mRNA vaccines are known to be powerful inducers of IFN-I (7). Therefore, the key question arises whether strong IFN-I production accounts for the side effects of COVID-19 vaccines? In considering this question, it should be noted that IFN-I has been used therapeutically for many years, currently for treating hepatitis $\mathrm{B}$ and $\mathrm{C}$ and multiple sclerosis. In these contexts, IFN-I injection elicits the same prominent pattern of fever, headaches and fatigue as the current COVID-19 vaccines. Moreover, when used repeatedly, therapeutic IFN-I administration can also lead to depression and cognitive slowing and thereby closely mimic the still-poorly understood clinical condition of chronic fatigue syndrome (8). Given that IFN-I stimulates synthesis of many different cytokines and chemokines, which of these downstream effects account for the symptoms of IFN-I administration is still unclear.

The notion that effective immune responses to SARS-CoV2 and other pathogens are IFN-I-dependent begs the question of how vaccines induce immunity. In addition to TCR recognition of antigen (MHC-associated peptides) on DC, T cells need a "second signal" to mount a productive immune response; this signal results from contact of T cell CD28 molecules with CD80 and CD86 molecules on DC. Without such costimulation, the $\mathrm{T}$ cell response may lead to tolerance rather than immunity. Hence, a mandatory feature of a successful vaccine is that, in addition to providing a source of antigen, the vaccine must contain an "adjuvant" in order to induce strong up-regulation of costimulatory molecules on host DC (9). Like IFN-I, adjuvants stimulate DC by binding to PRRs on these cells, which signals the cells to become activated and up-regulate costimulatory molecules. Many components of pathogens have adjuvant activity, notably mRNA and DNA. Moreover, adjuvant activity is conspicuous for Poly(I:C), a synthetic analog of double-stranded RNA; CpG oligodeoxynucleotides, which are short single-stranded synthetic DNA molecules; and Freund's Complete Adjuvant (CFA), a suspension of dried whole mycobacteria in mineral oil. Notably these and other nucleic acid-containing adjuvants are ineffective in $I F N A R^{-/-}$mice, indicating that these adjuvants operate by eliciting IFN-I production (10). Indeed, IFN-I itself is a powerful adjuvant.

From the above, it is highly likely - albeit not proven - that the side effects of COVID-19 vaccines are simply a by-product of a short burst of IFN-I generation concomitant with induction of an effective immune response. Notably, side effects vary considerably according to the recipient's age and sex, with more severe effects in females than males and in younger people than the elderly (11). The point to emphasize here is the striking correlation with IFN-I production. Thus, closely paralleling the intensity of typical immune responses, IFN-I generation is substantially stronger in females than males and in younger than older people.

For SARS-CoV-2 infection, it was mentioned earlier that IFN-I levels are low, reflecting antagonism by the virus. By contrast, IFN-I levels are generally high in influenza infection (3). This difference may explain why "flu-like" symptoms are prominent for influenza but usually mild during SARS-CoV-2 infection. It is worth noting, however, that current COVID-19 vaccines lead to selective expression of just the spike protein, which fails to antagonize IFN-I. Hence, IFN-I production by the vaccines might be appreciably higher than following SARS-CoV-2 infection itself, which could then explain why young people tend to have significant side effects to COVID19 vaccines yet can be asymptomatic during SARS-CoV-2 infection. Obtaining direct data on this issue is of obvious interest.

In light of the above, the prospect of fatigue and headache after vaccination for COVID-19 should be viewed positively: as a necessary prelude to an effective immune response. The side effects of vaccination will nearly always be mild and transient, and indicate merely that the vaccine is doing its job of stimulating production of interferon, the body's in-built immune stimulator.

\section{REFERENCES AND NOTES}

1. M. Wadman, Public needs to prep for vaccine side effects. Science 370, 1022 (2020). Medline

2. A. Remmel, COVID vaccines and safety: What the research says. Nature 590, 538540 (2021). doi:10.1038/d41586-021-00290-x Medline

3. G. Schreiber, The role of type I interferons in the pathogenesis and treatment of COVID-19. Front. Immunol. 11, 595739 (2020). doi:10.3389/fimmu.2020.595739 Medline

4. C. King, J. Sprent, Dual nature of type I interferons in SARS-CoV-2-induced inflammation. Trends Immunol. 42, 312-322 (2021). Medline

5. M. Contoli, A. Papi, L. Tomassetti, P. Rizzo, F. Vieceli Dalla Sega, F. Fortini, F. 
Torsani, L. Morandi, L. Ronzoni, O. Zucchetti, R. Pavasini, A. Fogagnolo, C. A. Volta, N. W. Bartlett, S. L. Johnston, S. Spadaro, G. Campo, Blood interferon- $\alpha$ levels and severity, outcomes and inflammatory profiles in hospitalized COVID-19 patients. Front. Immunol. 12, 648004 (2021). Medline

6. P. Bastard, L. B. Rosen, Q. Zhang, E. Michailidis, H. H. Hoffmann, Y. Zhang, K Dorgham, Q. Philippot, J. Rosain, V. Béziat, J. Manry, E. Shaw, L. Haljasmägi, P. Peterson, L. Lorenzo, L. Bizien, S. Trouillet-Assant, K. Dobbs, A. A. de Jesus, A. Belot, A. Kallaste, E. Catherinot, Y. Tandjaoui-Lambiotte, J. Le Pen, G. Kerner, B. Bigio, Y. Seeleuthner, R. Yang, A. Bolze, A. N. Spaan, O. M. Delmonte, M. S. Abers, A. Aiuti, G. Casari, V. Lampasona, L. Piemonti, F. Ciceri, K. Bilguvar, R. P. Lifton, M. Vasse, D. M. Smadja, M. Migaud, J. Hadjadj, B. Terrier, D. Duffy, L. Quintana-Murci, D. van de Beek, L. Roussel, D. C. Vinh, S. G. Tangye, F. Haerynck, D. Dalmau, J. Martinez-Picado, P. Brodin, M. C. Nussenzweig, S. Boisson-Dupuis, C. RodríguezGallego, G. Vogt, T. H. Mogensen, A. J. Oler, J. Gu, P. D. Burbelo, J. I. Cohen, A. Biondi, L. R. Bettini, M. D’Angio, P. Bonfanti, P. Rossignol, J. Mayaux, F. RieuxLaucat, E. S. Husebye, F. Fusco, M. V. Ursini, L. Imberti, A. Sottini, S. Paghera, E. Quiros-Roldan, C. Rossi, R. Castagnoli, D. Montagna, A. Licari, G. L. Marseglia, X. Duval, J. Ghosn, J. S. Tsang, R. Goldbach-Mansky, K. Kisand, M. S. Lionakis, A. Puel, S.-Y. Zhang, S. M. Holland, G. Gorochov, E. Jouanguy, C. M. Rice, A. Cobat, L. D. Notarangelo, L. Abel, H. C. Su, J.-L. Casanova; HGID Lab; NIAID-USUHS Immune Response to COVID Group; COVID Clinicians; COVID-STORM Clinicians; Imagine COVID Group; French COVID Cohort Study Group; Milieu Intérieur Consortium; CoV-Contact Cohort; Amsterdam UMC Covid-19 Biobank; COVID Human Genetic Effort, Autoantibodies against type I IFNs in patients with life-threatening COVID19. Science 370, 6515 (2020). doi:10.1126/science.abd4585 Medline
7. A. Cagigi, K. Loré, Immune responses induced by mRNA vaccination in mice, monkeys and humans. Vaccines (Basel) 9, 1-14 (2021). doi:10.3390/vaccines 9010061 Medline

8. A. Russell, N. Hepgul, N. Nikkheslat, A. Borsini, Z. Zajkowska, N. Moll, D. Forton, K. Agarwal, T. Chalder, V. Mondelli, M. Hotopf, A. Cleare, G. Murphy, G. Foster, T. Wong, G. A. Schütze, M. J. Schwarz, N. Harrison, P. A. Zunszain, C. M. Pariante, Persistent fatigue induced by interferon-alpha: A novel, inflammation-based, proxy model of chronic fatigue syndrome. Psychoneuroendocrinology 100, 276285 (2019). doi:10.1016/i.psyneuen.2018.11.032 Medline

9. S. G. Reed, M. T. Orr, C. B. Fox, Key roles of adjuvants in modern vaccines. Nat. Med 19, 1597-1608 (2013). doi:10.1038/nm.3409 Medline

10. E. Proietti, L. Bracci, S. Puzelli, T. Di Pucchio, P. Sestili, E. De Vincenzi, M. Venditti, I. Capone, I. Seif, E. De Maeyer, D. Tough, I. Donatelli, F. Belardelli, Type I IFN as a natural adjuvant for a protective immune response: Lessons from the influenza vaccine model. J. Immunol. 169, 375-383 (2002). doi:10.4049/iimmunol.169.1.375 Medline

11. M. J. Bunders, M. Altfeld, Implications of sex differences in immunity for SARSCoV-2 pathogenesis and design of therapeutic interventions. Immunity 53, 487495 (2020). doi:10.1016/j.immuni.2020.08.003 Medline

Acknowledgments: Funding: This work was supported by grant APP1145714 from National Health and Medical Research Council (NHMRC, Australia) to J.S. and by grants NHMRC APP2004306 and Australian Research Council DP210103811 to C.K. Author contributions: J.S. and C.K. wrote the manuscript. Competing interests: The authors declare that they have no competing interests.

Published First Release 22 June 2021

10.1126/sciimmunol.abj9256 


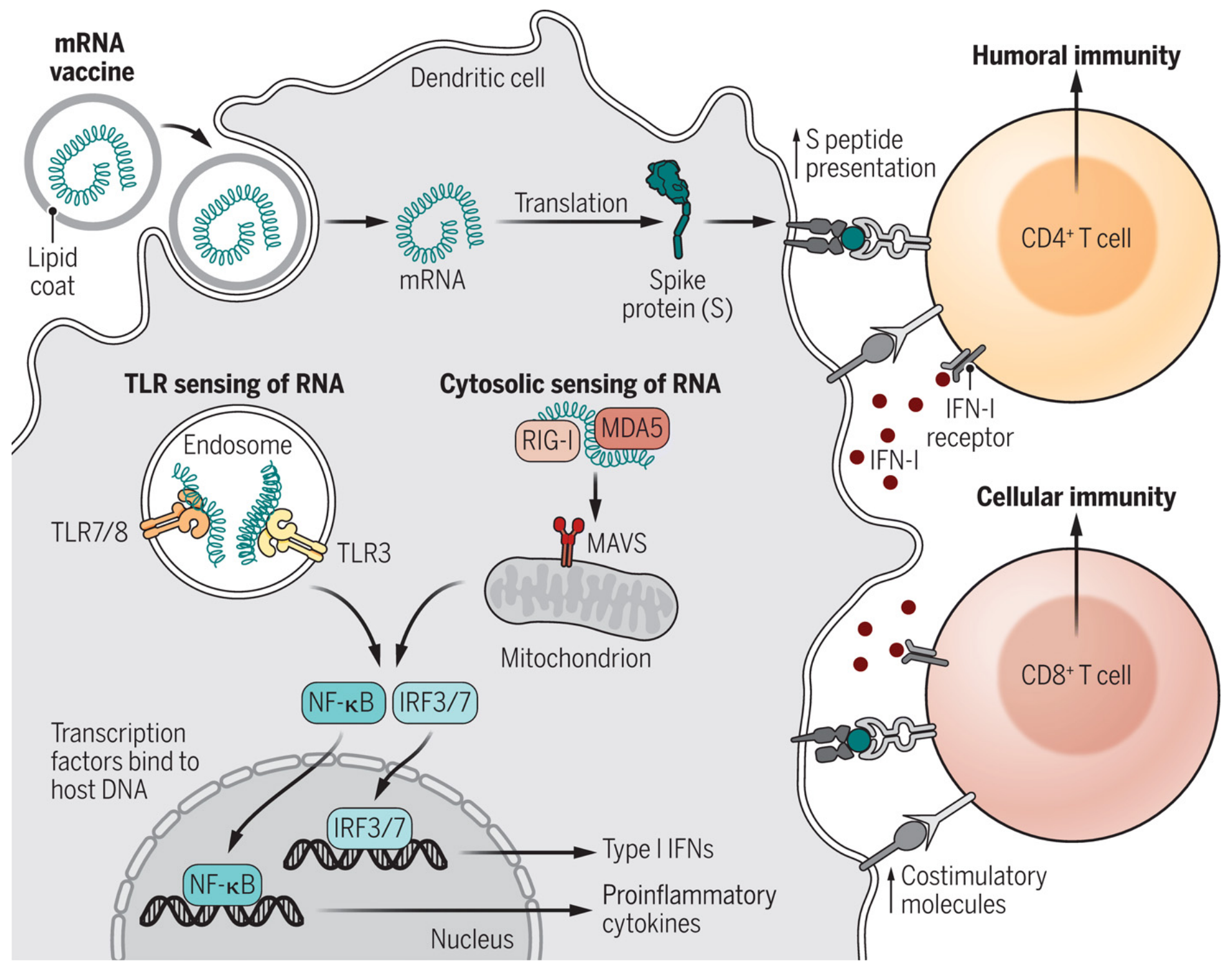

Fig. 1. mRNA vaccine activation of DC and induction of type I interferon. After uptake, mRNA is translated into spike protein and presented as cell-surface $\mathrm{MHC}$-bound peptides to $\mathrm{CD} 4^{+}$and $\mathrm{CD} 8^{+} \mathrm{T}$ cells. Cytosolic sensing of RNA by RIG-I and MDA-5 plus TLR binding within endosomes leads to activation of IRF3/7 and NF-kB, which bind to DNA inducing gene transcription, and production of IFN-I and pro-inflammatory cytokines, respectively. Through up-regulation of DC costimulatory molecules, production of stimulatory cytokines, and a direct action on T cells, IFN-I guides and promotes the adaptive immune response of T and B cells. 